\title{
Band Analysis for Land Use in Multi Spectral Images
}

\author{
Hidayat Ur Rahman \\ Department of Computer \\ Systems Engineering UET \\ Peshawar Pakistan
}

\author{
Nasru Minallah \\ Department of Computer \\ Systems Engineering UET \\ Peshawar Pakistan
}

Rehanullah Khan

Department of Information Technology, Qassim University, Al-Qassim, KSA

\author{
Ali Alkhalifah \\ Department of Information \\ Technology, Qassim University, \\ Al-Qassim, KSA
}

\begin{abstract}
Hyper spectral and multi spectral image analysis is the commonly used technique for land use and land cover classification. Effective use of the land cover can play a vital role in the development of country. Multi spectral satellites use passive sensor, hence the only source of energy involved in the acquisition of satellite imagery is the reflectance of the sun. In order to investigate the role of individual bands of the Visible and infra-red region in the recognition of land covers such as vegetation, non-vegetation, settlements and barren land an extensive research has been carried out.

This paper is focused in the dissection and contribution of individual component (band) of SPOT-5 imagery for land cover analysis as well. In this article extensive experimentation has been carried out which reveals the effect of individual and combine bands in the recognition of land cover. Classifications of various bands were done using supervised machine learning classification, random forest classifier has been used for classification purpose.
\end{abstract}

\section{Keywords}

Land cover classification, SPOT-5, multi-spectral imagery, random forest, NIR, SWIR

\section{INTRODUCTION}

In remote sensing analysis, the primary source of obtaining the imagery is satellite. The sensors in the satellite are responsible for the conversion of sunlight energy into pixels. Based on the sensor features sensors are either multi spectral or hyper spectral. Multi spectral sensors has multiple bands while hyper spectral has more than hundred bands and are thus hyper spectral sensors are more powerful from multispectral with the capability of storing more information regarding the geography of a surface.

To make use of the satellite imagery commonly machine learning techniques are used. Common approaches followed are pixel and object based classification of the imagery. In object based classification approach, the given image is divided into small object called patches, based on patches training set is created for classifier learning while in pixel based approach the underlying pixels of the image is considered as features, these features are processed for the detection of area of interest. Various methods has been adopted for the classification of land covers, in literature the state of art classifier used for land cover analysis, change detection and land use analysis are SVM, ANN, MLE and Random forest. Maximum likelihood classifier is from the parametric set of classifiers which use the prior information about the event. Based on these prior probabilities the probability of an event for all the classes are calculated, the most probable class for the event is the one having the maximum probability. The performance of maximum likelihood compared with the distance based classifier such as Minimum distance and Mahalanobis distance, which showed that maximum likelihood has the high recognition compared to the other classifiers, the comparison was carried out on raw Landsat thematic Mapper imagery having resolution of $90 \mathrm{~m}$ [1]. For pixel based classification such as change analysis, land cover classification is mostly suited [3-6]. The strength of MLE has also been investigated in the use of thematic data [2]. MLE uses the probability distribution based on which the incoming pixel is classified and hence this technique fails to discriminate between pixels having the same spectral signatures compared to non-parametric classifier such as ANN [7]. A neural network consists of input, output and hidden layer. The input layer of the neural network consist of raw data which is presented in the form of vectors, the hidden layer contains the kernels or activation function which maps the input to the output layer. The strength of ANN has influenced the process of classification of both hyper spectral and multi-spectral satellite imagery compared to other parametric approaches [8-12]. Beside ANN and MLE SVM is also used for land cover classification. On low resolution satellite imagery SVM has outperformed MLE and ANN [13]. SVM crates an optimal decision boundary for the classes, originally the SVM were used for binary classification. Today SVM has various nonlinear kernels such as Basis function (RBF), Polynomial and sigmoid [14]. For pixel based classification of satellite imagery parametric and nonparametric classifier has been used [15]. Based SVM and ANN, Random forest is also used for land cover analysis in the recent studies [18]. A random forest is a tree based classifier or more specifically random forest is an ensemble classifier which combines more than one tree using bootstrapping and bagging approaches to make a random forest. More detail about random forest can be found in [17]. The comparison between parametric random forest and nonparametric MLE shows that random forest has the high accuracy on high resolution imagery [18]. The random forest based approach has also been applied to the high resolution Landsat Imagery and has shown better results [19]. For agricultural change detection the use of Normalized difference vegetation Index (NDVI) has been advocated. NDVI uses vegetation indexes which are helpful in determining the change in the agriculture. In [16], the author has demonstrated the role of vegetation indexes to locate the land use and land cover changes over a period of time.

In this paper, we have illustrated the role of individual band of the multi-spectral satellite imagery on land use classification detection and classification. The experimentation setup was carried out on SPOT-5 satellite imagery which consist of Four bands namely Green, Red, Near infra-red and Short Wave Infra-red. The high resolution dataset were classifier using 10 
Fold cross validation. For the purpose of classification we have used the random forest based approach which has shown better accuracy for high resolution imagery from the state of the art.

\section{DATASET DESCRIPTION}

The dataset we have used in this experiment is obtained from SPOT-5 satellite. SPOT-5 consist of four individual bands each having resolution of $2.5 \mathrm{~m}$. These Four bands are Green, Red, near infra-red and Short wave infra-red. Samples from each band of the imagery are collected as shown in Figure-1. From Figure-1 we see that our ROI's consists of six classes. Water bodies consist of lakes, rivers, channels and used water. The deserts contain the land which is not in use especially, for the agricultural purpose. Other vegetation category consists of fruits such as leeches, peaches and persimmons. Settlement contains the land which is used in homes, buildings, hospital and schools etc. The other two categories consist of Tobacco and Sugar crop. A total of 16625 instances were used which is distributed as shown below in Figure 1.

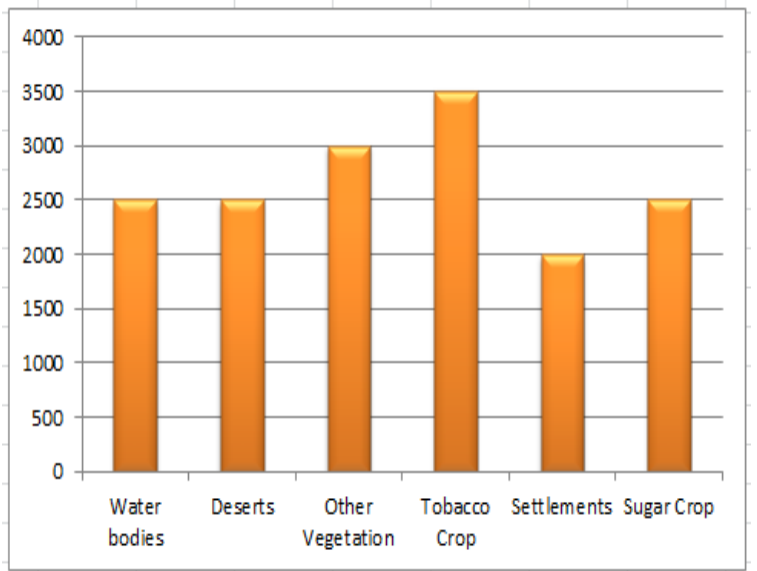

Figure-1: Pixel distribution for ROI's

\section{METHODOLOGY}

Our approach of investigating the individual band spectral reflectance toward a particular land cover is based on machine learning approach. The spectral reflectance of a band shows how much information particular components carry about the ROI. In this extensive study we have indicated the individual role of each component and also there combine efforts in the recognition of Land covers. Our experimental setup comprises of SPOT-5 dataset which contains of four bands. I.e. Green, Red, NIR and SWIR as discussed before. Individual and combine effect of the each band were investigated using random forest classifier.

We have selected the random forest approach based on overall good performance in our test analysis. The Random forest combines decision trees. A random forest contains more than two trees; the upper limit can be set by the user. Random forest learning occurs when a random vector is produced equal to the size of the number of trees. Using these vectors, the trees are trained [18]. Random vectors are distributed in such a way that they are independent of each other. To classify the incoming pixel into a class, a voting amongst the trees is done. Let suppose, a random vector $\theta_{n}$ having size of $\mathrm{n}$, where $\theta_{n}=\left\{\theta_{1}, \theta_{2} \ldots \ldots . \theta_{n}\right\}$ [18] and tree ensembles $h_{k}(x)=\left\{h_{1}(x), h_{2}(x) \ldots \ldots \ldots h_{k}(x)\right\}$ [18]. After training the classifiers on the random vector, we obtain $\mathrm{h}\left(\mathrm{x}, \theta_{n}\right)$. Here we can see that each random vector is used to classify the input pixels, after classifying the pixels, a voting amongst the tree is done to select the most favorable class. Class having the highest vote for a pixel is assigned to that class. In our experiment, we have used ten trees with a seed of 1 having depth of 0.Using the random forest classifier we have measured the Precision recall and F-score of the spectral features in the representation of ROI.

\section{RESULTS AND DISCUSSION}

For independent band role analysis we have used multispectral imagery, details about the imagery is provided in dataset details section. The experimental setup was performed using 10 Fold cross validation paradigm on the six classes of dataset as shown in Figure 1. The random forest classifier was selected due to its over-all good performance on this dataset. For the performance evaluation of the different possibilities, we use F-score (in percentage) which equates to an average of precision and recall. Table 1 shows the F-score computed for the individual bands i.e. Green, Red, NIR and SWIR for all of the six classes. From Table-1 we noticed that the highest Fscore of 56 has been observed for Water bodies in the NIR, while lowest F-score of 7 in observed in the SWIR. Deserts are best visible in the SWIR band while Red and NIR infrared band relative low visibility. SWIR has the best F-score in the detection of sparse vegetation and settled areas as shown in Table-1. Tobacco is best represented in the NIR while sugar cane has spectral reflection in the Red band having Fscore of 74

Table-1: F-measure (in percent) for six classes

\begin{tabular}{|lllll|}
\hline & Green & Red & NIR & SWIR \\
\hline Water Bodies & 54 & 52 & $\mathbf{5 6}$ & 07 \\
\hline Deserts & 32 & $\mathbf{2 8}$ & $\mathbf{2 8}$ & $\mathbf{3 5}$ \\
\hline Other Veg. & 53 & $\mathbf{5 1}$ & 48 & $\mathbf{8 0}$ \\
\hline Tobacco Crop & 32 & 42 & $\mathbf{4 4}$ & $\mathbf{1 8}$ \\
\hline Settlement & $\mathbf{4 6}$ & 64 & 60 & $\mathbf{8 0}$ \\
\hline Sugar Crop & $\mathbf{2 4}$ & $\mathbf{7 4}$ & 69 & 60 \\
\hline
\end{tabular}

The results in Table 1 show that individual band cannot accurately represent the different land covers although it helps in investigating the visible and infra-red bands for the representation of Vegetation, Non vegetation and urban areas.

For more analysis, we proceed by merging different bands as shown in Table 2. The merging of the two bands emerges as different combination pairs. From the Table 2, the highest Fscore reported for Water is 85 , obtained by the combination of NIR and SWIR and the lowest of 67 by Green with the SWIR. For deserts, the highest f-score of 89 is reported for R and SWIR and the lowest of 49 for $\mathrm{R}$ and NIR. For other vegetation, the fusion of NIR and SWIR shows the highest $\mathrm{f}$ score of 78 , while G+NIR, G+NIR and R+NIR pairs show considerably low f-score of 66 . Sugar cane has relatively low detection in the fusion of R and NIR pair. Tobacco has highest F-score of 83 in the Green and SWIR while having low detection in the Red and SWIR region. The settlement class is more prominent in the visible spectrum i.e. Red and Green while having poor detection in the Green and NIR region. 
Table 2: The table show the F-score (in percent) measured for the fusion of two bands for each class

\begin{tabular}{|c|c|c|c|c|c|c|}
\hline 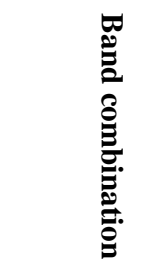 & 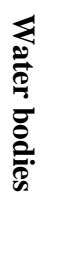 & 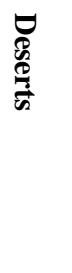 & 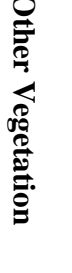 & 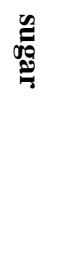 & 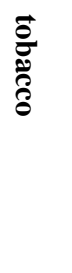 & 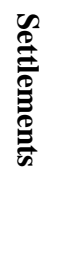 \\
\hline $\mathbf{G}+\mathbf{R}$ & 77 & 73 & 66 & 66 & 80 & 89 \\
\hline G+NIR & 73 & 69 & 66 & 66 & 75 & 79 \\
\hline G+SWIR & 67 & 70 & 67 & 65 & 83 & 82 \\
\hline $\mathbf{R}+\mathrm{NIR}$ & 83 & 49 & 66 & 54 & 81 & 88 \\
\hline R+SWIR & 77 & 89 & 74 & 56 & 74 & 80 \\
\hline $\begin{array}{l}\text { NIR+SWI } \\
\text { R }\end{array}$ & 85 & 86 & 78 & 56 & 76 & 85 \\
\hline
\end{tabular}

From Table-2 we have measured F-score greater than 80 for more than two cases. By combining more than 2 bands better analysis about the spectral reflectance can be achieved. For this purposed we studied the combining effect of three and four bands.

Table 3 compares three bands vs. four bands. We represent the fusion of four bands as the benchmark for the comparison. From the Table 3, the highest F-measure (last row in Table 3) for each of these categories is observed in the combination of visible and infra-red bands as a pair $\mathrm{R}+\mathrm{G}+\mathrm{NIR}+\mathrm{SWIR}$. For the three pairs, the water have the $2^{\text {nd }}$ highest F-score of 88 in the Red, NIR and SWIR pair, while, visible and NIR shows the lowest detection of 85. The combination of Green, red and NIR shows the lowest prediction for all the classes except settled areas as shown in bold italic (first row) in Table 3 .

Table 3: F-score (in percent) achieved by the random forest classifier with the fusion of three and four bands for land use analysis

\begin{tabular}{|c|c|c|c|c|c|c|}
\hline 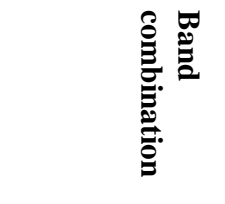 & 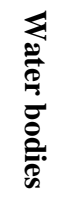 & 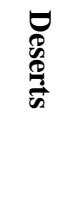 & 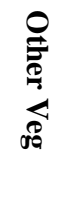 & 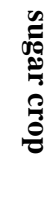 & 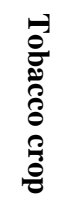 & 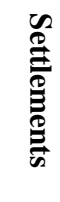 \\
\hline G+R+NIR & 85 & 79 & 76 & 69 & 86 & 91 \\
\hline G+NIR+SWIR & 88 & 89 & 83 & 77 & 90 & 92 \\
\hline G+R+SWIR & 87 & 89 & 79 & 74 & 87 & 93 \\
\hline R+NIR+SWIR & 88 & 89 & 82 & 71 & 88 & 90 \\
\hline $\begin{array}{l}\mathrm{R}+\mathrm{G}+\mathrm{NIR}+\mathrm{SW} \\
\text { IR }\end{array}$ & 90 & 91 & 84 & 78 & 91 & 93 \\
\hline
\end{tabular}

For Deserts, the f-score reported for all the possible combinations is almost the same with a slight variation. The Vegetation class has highest detection in the Green, NIR and SWIR. Similarly, the sugarcane and tobacco follows the same trend. Settlements, has strong detection performance in the Green, Red and SWIR.

Based on the extensive experimentation, we deduce that the addition of extra bands helps in building a robust detection model and that land cover is best represented by the combination of visible and infra-red bands: Green, Red, NIR and SWIR.

For further elaboration, we show combined F-score of all the classes for using the three and four bands as shown in Figure2. From Figure 2, we note that the combination of Green, NIR and SWIR results in overall F-score of 86 , this is $2 \%$ less compared to the combination of Red, Green, NIR and SWIR which is $88 \%$. This reveals that only $2 \%$ information is presented in Red band. The combination of Green, Red and NIR has the lowest f-measure of $83 \%$. Other band combination such as Green, Red and SWIR performed better compared to $\mathrm{G}+\mathrm{R}+\mathrm{NIR}$.

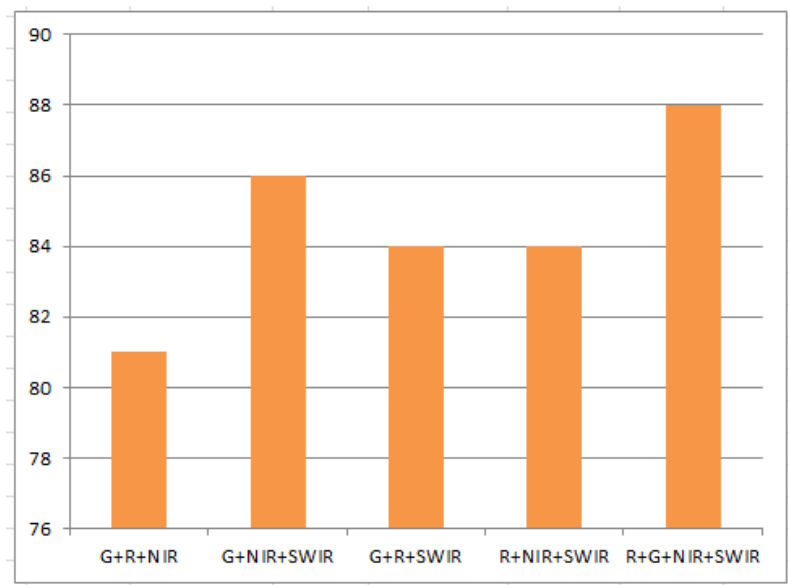

Figure 2: the combined F-score for the all the classes using three and four bands pairs.

Based on the results obtained in table 1,2 and 3 we are able to deduce the role of each independent bands for land cover classification. From Figure-2 we can deduce that only 2 percent distinct information is carried in the Red band. The visible region i.e. red and green band is best suited for the recognition of settled areas, while for water bodies' best analysis is done in the infra-red regions. In our experiment we have used three categories for vegetation i.e. Tobacco crop which is best viewed in the Green and infra-red regions and very little information is stored in the red band. Sparse vegetation and sugar cane is best represented in the infra-red and green region. in short we have concluded that infra-red region is favored for the detection of vegetation and nonvegetation land covers while visible regions provides very little information about these categories.

\section{CONCLUSION}

In this article, based on independent and fusion of bands, we have analyzed the visible and infra-red bands of the Electromagnetic spectrum for the analysis of Water bodies, Deserts, Tobacco, sugar cane, other vegetation and settlements. We have shown that the Electromagnetic spectrum can be best utilized for land use analysis by combining the visible and infra-red spectrum. We have further shown that the infra-red spectrum is best suited for the 
detection of vegetation and non-vegetation areas while the visible spectrum carries less information and has only good detection in the classification of settled areas. The red band in the spectrum provides only $2 \%$ information of the visible and infra-red region and hence cannot differentiate between different spectral signatures.

\section{REFERENCES}

[1] F.S.Al-Ahmadi and A.S Hames.2009 "Comparison of Four Classification Methods to Extract Land Use and Land Cover from Raw Satellite Images for Some Remote Arid Areas, Kingdom of Saudi Arabia”. Journal of King Abdulaziz University-Earth Sciences Vol. 20(1).. .

[2] Jensen, J.R. Thematic information extraction: pattern recognition. In Introductory Digital Image Processing A Remote Sensing Perspective, 3rd ed.

[3] Keith, C.C., Ed.; 2005 Prentice Hall Series in Geographic Information Science: Saddle River, NJ, USA, pg- 337-406.

[4] Currit, N.2005 Development of remotely sensed, historical land cover change database for rural Chihuahua, Mexico. Int. J. Appl. Earth Obs. Geoinf. Vol7, 232-247.

[5] Bailly, J.S.; Arnaud, M.; Puech, C. Boosting: 2007 a classification method for remote sensing. Int. J. Remote Sens. Vol-28, pg-1687-1710.

[6] Liu, X.H.; Skidmore, A.K.; Oosten, H.V.2002 Integration of classification methods for improvement of land cover map accuracy. ISPRS J. Photogramm. Remote Sens. Vol-56, pg-257-268.

[7] Weng, Q. 2002 Land use change analysis in the Zhujiang Delta of China using satellite remote sensing, GIS and stochastic modeling. J. Environ. Manage.Vol-64, 273284

[8] F. sunarErbek, C.Ozkan and M.Taberner. jan 2004 "Comparison of maximum likelihood classification method with supervised artificial neural network algorithms for land use activities". International Journal of Remote sensing.Vol. 25(9), Pg 1733-1748.

[9] Verbeke, L.P.C.; Vancoillie, F.M.B.; De Wulf, R.R.2004 Reusing back-propagation artificial neural networks for land cover classification in tropical savannahs. Int. J. Remote Sens. Vol-25, 2747-2771.

[10] Lee, J.;1990 A neural network approach to cloud classification. IEEE Trans. Geosci. Remote Sens. Vol$28,846-855$.

[11] Baraldi, A.; Parmiggiani, F. 1995 A neural network for unsupervised categorization of multivalued input patterns: an application to satellite image clustering. IEEE Trans. Geosci. Remote Sens. Vol- 33, 305-316.

[12] Bischof, H.; Leonardis, 1998 A. Finding optimal neural networks for land use classification. IEEE Trans. Geosci. Remote Sens. Vol-36, 337-341.

[13] Serpico, S.B.; Roli, F.1995 Classification of multisensor remote-sensing images by structured neural networks. IEEE Trans. Geosci. Rem. Sens. Vol-33, 562-578.

[14] Huang, C., Davis, L.S., Townshed, J.R.G. (2002). An assessment of support vector machines for land cover lassification. International Journal of Remote Sensing, $23,725-749$.

[15] Haykin, S. (1999) Neural Networks: A Comprehensive Foundations, 2ed. Upper Saddle River: Prentice Hall.

[16] Ehsan Sahebjalal1 and Kazem Dashtekian. 26 September 2103 "Analysis of land use-land covers changes using normalized difference vegetation index (NDVI) differencing and classification methods". African Journal of Agricultural Research Vol. 8(37)..

[17] Lyon JG, Yuan D, Lunetta RS (1998). A change detection experiment using vegetation indices. Photogramm. Eng. Rem. Sens. 64(2):143150.

[18] LeoBreiman,2001 "Randomforests," Mach.Learn., vol.45, no. 1 , pp. 5-32.

[19] Matthew M.Hayes,Scott N.Miller, Melanie A.Murphy. 2014 "High-resolution landcover classification using Random Forest". Remote sensing letters Vol.5

[20] V.F. Rodriguez-Galiano, B. Ghimire, J. Rogan, M Chica-Olmo, J.P. Rigol-Sanchez January 2012“An assessment of the effectiveness of a random forest classifier for land-cover classification". ISPRS Journal of Photogrammetry and Remote Sensing, Volume 67, Pages 93-104 